\title{
Correction to: Computational approaches for cancer 2017 workshop overview
}

Sunita Chandrasekaran ${ }^{1}$ and Eric Stahlberg ${ }^{2^{*}}$

Correction to: BMC Bioinformatics 2018, 19

(Suppl 18): 487.

https://doi.org/10.1186/s12859-018-2502-x

It was highlighted that the original article [1] contained errors.

The list of authors and submissions for the supplement contained some outdated information and could be improved for readability and clarity. Below is the reformatted and corrected list.

CAT: computer aided triage improving upon the Bayes risk through $\varepsilon$-refusal triage rules

Nicolas Hengartner, Leticia Cuellar, Xiao-Cheng Wu, Georgia Tourassi, John Qiu, Blair Christian and Tanmoy Bhattacharya.

Sparse coding of pathology slides compared to transfer learning with deep neural networks

Will Fischer, Sanketh S. Moudgalya, Judith D. Cohn, Nga T. T. Nguyen and Garrett T. Kenyon.

Real-time data analysis for medical diagnosis using FPGA-accelerated neural networks

Ahmed Sanaullah, Chen Yang, Yuri Alexeev, Kazutomo Yoshii and Martin C. Herbordt.

High-throughput binding affinity calculations at extreme scales

Jumana Dakka, Matteo Turilli, David W. Wright, Stefan J. Zasada, Vivek Balasubramanian, Shunzhou Wan, Peter V. Coveney and Shantenu Jha.

Deep clustering of protein folding simulations

Debsindhu Bhowmik, Shang Gao, Michael T. Young and Arvind Ramanathan.
CANDLE/Supervisor: a workflow framework for machine learning applied to cancer research

Justin M. Wozniak, Rajeev Jain, Prasanna Balaprakash, Jonathan Ozik, Nicholson T. Collier, John Bauer, Fangfang Xia, Thomas Brettin, Rick Stevens, Jamaludin Mohd-Yusof, Cristina Garcia Cardona, Brian Van Essen and Matthew Baughman.

Predicting tumor cell line response to drug pairs with deep learning

Fangfang Xia, Maulik Shukla, Thomas Brettin, Cristina Garcia-Cardona, Judith Cohn, Jonathan E. Allen, Sergei Maslov, Susan L. Holbeck, James H. Doroshow, Yvonne A. Evrard, Eric A. Stahlberg and Rick L. Stevens.

High-throughput cancer hypothesis testing with an integrated PhysiCell-EMEWS workflow

Jonathan Ozik, Nicholson Collier, Justin M. Wozniak, Charles Macal, Chase Cockrell, Samuel H. Friedman, Ahmadreza Ghaffarizadeh, Randy Heiland, Gary An and Paul Macklin.

Scalable deep text comprehension for Cancer surveillance on high-performance computing

John X. Qiu, Hong-Jun Yoon, Kshitij Srivastava, Thomas P. Watson, J. Blair Christian, Arvind Ramanathan, Xiao C. Wu, Paul A. Fearn and Georgia D. Tourassi.

\section{Author details}

${ }^{1}$ Department of Computer and Information Sciences, University of Delaware, Newark, DE, USA. ${ }^{2}$ Biomedical Informatics and Data Science Directorate,

Frederick National Laboratory, Frederick, MD, USA.

Published online: 22 January 2019

Reference

1. Chandrasekaran and Stahlberg. Computational approaches for Cancer 2017 workshop overview. BMC Bioinformatics. 2018;19(Suppl 18):487. https://doi. org/10.1186/s12859-018-2502-x.

\footnotetext{
* Correspondence: eric.stahlberg@nih.gov

${ }^{2}$ Biomedical Informatics and Data Science Directorate, Frederick National

Laboratory, Frederick, MD, USA

Full list of author information is available at the end of the article
} 